\title{
Monitoring of rhythms in laser speckle data
}

\author{
D. E. Postnov*, A. Y. Neganova and D. D. Postnov \\ Physics Department, Saratov State University \\ Astrakhanskaya St. 83, Saratov 410012, Russia \\ *postnov@info.sgu.ru \\ A. R. Brazhe \\ Biophysics Department, Biological Faculty, Moscow State University \\ Leninskie Gory 1, Building 12, 119991 Moscow, Russia
}

Received 15 July 2013

Accepted 15 November 2013

Published 2 January 2014

\begin{abstract}
While the laser speckle imaging (LSI) is a powerful tool for multiple biomedical applications, such as monitoring of the blood flow, in many cases it can provide additional information when combined with spatio-temporal rhythm analysis. We demonstrate the application of Graphics Processing Units (GPU)-based rhythm analysis for the post processing of LSI data, discuss the relevant structure of GPU-based computations, test the proposed technique on surrogate 3D data, and apply this approach to kidney blood flow autoregulation. Experiments with surrogate data demonstrate the ability of the method to extract information about oscillation patterns from noisy data, as well as to detect the moving source of the rhythm. The analysis of kidney data allow us to detect and to localize the dynamics arising from autoregulation processes at the level of individual nephrons (tubuloglomerular feedback (TGF) rhythm), as well as to distinguish between the TGF-active and the TGF-silent zones.
\end{abstract}

Keywords: Laser speckle imaging; wavelet transform; kidney autoregulation.

\section{Introduction}

Laser speckle imaging (LSI) is one of the rapidly developing optical techniques. In biomedical applications, LSI is used for measuring tissue perfusion rate, ${ }^{1,2}$ or the blood flow rate in small vessels. ${ }^{3,4}$
For research and diagnostic purposes, it is useful not only to perform local measurement, but also to obtain temporal and spatial patterns of the blood flow. There are examples of successful LSI application for measuring spatial and temporal hemodynamic alterations in microvascular networks. ${ }^{5,6}$

\footnotetext{
${ }^{*}$ Corresponding author.
}

This is an Open Access article published by World Scientific Publishing Company. It is distributed under the terms of the Creative Commons Attribution 3.0 (CC-BY) License. Further distribution of this work is permitted, provided the original work is properly cited. 
The LSI-based studies on cerebral blood flow also utilize the ability of the method to deliver the information about non-stationary, time varying and spatially inhomogeneous processes. ${ }^{7-9}$

In the above examples, the useful information was obtained by means of calculation of time- and/ or space-averaged speckle contrast pattern or relative slow blood flow changes. However, in many cases rhythmic changes of flow rate (not flow rate itself) within specific frequency range can provide useful information.

Vasomotion is a physiological process in which blood vessels spontaneously and rhythmically dilate and constrict with frequency from 0.015 to $0.3 \mathrm{~Hz} \cdot{ }^{10,11}$ The underlying mechanism is based on ionic mechanisms in vascular smooth muscle cells. ${ }^{12,13}$ Additional experimental verification of predictions made on temporal and spatial features of such self-sustained behavior of arterial wall is needed.

Experimental studies on kidney blood flow autoregulation show the existence of pronounced rhythmic component in the proximal intratubular pressure of individual nephron. ${ }^{14}$ This rhythm appears due to the so-called tubuloglomerular feedback (TGF) and reflects the essential feature of nephrons (and, hence, kidney) functioning. It was shown that the proximal tubular pressure, distal tubular $\mathrm{Cl}^{-}$concentration and single nephron blood flow dynamics exibit typical features of self-sustained oscillations. ${ }^{15,16}$ Attempts to understand the behavior of large nephron ensembles ${ }^{17,18}$ reveal the need for information on rhythmic components of renal blood flow. Recently, LSI was used to quantify synchronization of TGF-oscillations between many nephrons identified on the kidney surface. ${ }^{6}$

We suggest that LSI technique can gain from the combination with advanced data analysis techniques. Windowed Fourier transform ${ }^{19,20}$ or wavelet transform ${ }^{19,21}$ can be used to extract additional information about rhythmic activity. However, there are some computational issues.

First, the LSI data are typically recorded as a sequence of frames. Thus, to obtain the single time course in selected location one needs to pass through all frames. It implies either to load all data in computer RAM, which is memory expensive, or to build a time course from file(s) stored on the disk, which is slow. Second, the spectral analysis over all frames requires much more computational power than calculation of speckle contrast and/or flow rate from the original data.
In the recent years, the rapidly developing GPUbased parallel computing technique ${ }^{22,23}$ provided an effective and relatively inexpensive solution for the second mentioned problem. GPU-based computing has recently been applied in optical coherence tomography (SS-OCT) systems,${ }^{24}$ as well as for real-time processing of raw speckle data. ${ }^{25,26}$

Here, we demonstrate the application of GPUpowered rhythm analysis for the post-processing (further analysis of already calculated relative flow rate) of LSI data. We discuss the relevant structure of GPU-based computations, test the proposed technique on surrogate 3D data, and illustrate its application to the study of autoregulation of kidney blood flow.

\section{Methods and Materials}

\subsection{Surrogate data}

In order to verify the performance of the proposed computational schemes, the surrogate data are prepared in the form of frame sequences. Each frame represents a predefined pattern obtained by mixture of noise and periodic signals. Namely:

- For the simulation of a spatially stable oscillating pattern, surrogate data consisted of 1000 frames each of $100 \times 100$ pixels. Multimodal temporal dynamics is simulated using six harmonic signals of unit amplitude with frequencies $1 / 6,1 / 18$, $1 / 25,1 / 30,1 / 100,1 / 23 \mathrm{~Hz}$, and the spatially and temporary uncorrelated noise with uniformly distributed values. The sum of all harmonic signals is biased to be always positive and then the noise term is added.

- To simulate a moving oscillating object, the number of frames is increased to 2500 with the same temporal structure. The spatial pattern is the spot of $15 \times 15$ pixels size moving from top left to bottom right corners.

\subsection{Kidney blood flow data}

Experimental LSI data of kidney blood flow in rats were provided by Holstein-Rathlou and Sosnovtseva, Univ. of Copenhagen. Physiological interpretations as well as animal preparation are described in Ref. 6 . The measurements were made using speckle contrast imager "moorFLPI" (Moor Instruments, UK) in high spatial resolution mode. The raw speckle data were averaged in time domain over 25 frames in order to 
calculate single frame of flow rate. The resulted data contain 2640 frames with resolutions of $760 \times 568$ pixels each. Resulted sampling rate is 1 frame per second, so the total recording time is $44 \mathrm{~min}$. Since we focus on the frequency range below the $0.03 \mathrm{~Hz}$, such value of sampling rate provides more then 30 points per period. It means that the time domain averaging of raw data does not limit our computations.

\subsection{Data analysis}

Data can be viewed as 3D array and has the form $d(x, y, l)$, where induces $x$ and $y$ denote the pixel position within the frame, and $l$ is the discrete time (frame number). The actual time $t=\tau l$, where $\tau$ is the sampling time interval.

Conventional discrete windowed Fourier transform (WFT) of $1 \mathrm{D}$ data can be described by the following equation ${ }^{19}$ :

$$
S(m, l)=\sum_{n=0}^{N-1}\left(d(n) g(n-m) e^{-j \frac{2 \pi l n}{N}}\right),
$$

where $g(n-m)$ is Fourier window. Because we use rectangle window $g(t)=1,{ }^{19}$ and since we actually have a $2 \mathrm{D}$ array of $1 \mathrm{D}$ windowed Fourier transforms, Eq. (1) takes the following form:

$$
S(x, y, k, l)=\sum_{n=k \cdot N}^{(k+1) N-1}\left(d(x, y, n) e^{-j \frac{2 \pi l n}{N}}\right),
$$

where $k$ denotes window number, and $N$ is the point number in each window. Actual frequency values are calculated as $f=l /(2 N \tau)$. Each value of $k$ corresponds to the time interval $t \in(k N \tau$, $(k+1) N \tau)$. Since the experimental data is a random process, throughout this paper we use the power spectrum obtained from the complex result of Fourier transform as $|S|^{2}$, where $|\cdot|$ means the modulus of the complex quantity.

The power spectrum of all data is obtained by averaging of $|S|^{2}$ over the $N_{k}$ data windows:

$$
\overline{|S(x, y, l)|^{2}}=\frac{1}{N_{k}} \sum_{k=0}^{N_{k}}|S(x, y, k, l)|^{2} .
$$

Continious wavelet transform (WT) based on the Morlet wavelet is used in the following form ${ }^{19}$ :

$$
W(x, y, a, l)=\frac{1}{\sqrt{|a|}} \sum_{k=0}^{N-1} d(x, y, l) e^{j 2 \pi k_{0} \frac{k-l}{a}} e^{\frac{-\left(\frac{k-l}{a}\right)^{2}}{2}},
$$

where $l$ is the number of sample, $k_{0}$ is the parameter, $a$ is the scaling parameter. As above, the actual time is $t=\tau l$, while the frequency can be estimated as $f \approx 1 / a \tau$ for $k_{0} \approx 1$. Similar to WFT, wavelet power spectrum is calculated as $|W|^{2}$.

Both Fourier, and wavelet power spectra can be averaged over some part of the frame, or over the whole frame:

$$
\begin{aligned}
|S|_{A}^{2}(k, l) & =\frac{1}{N_{A}} \sum_{x, y \in A}|S(x, y, k, l)|^{2}, \\
|W|_{A}^{2}(a, l) & =\frac{1}{N_{A}} \sum_{x, y \in A}|W(x, y, a, l)|^{2},
\end{aligned}
$$

where $A$ denotes some area less or equal to the whole frame, and $N_{A}$ is the number of pixels within $A$.

Throughout the paper, we use space-averaged spectra according to Eq. (5), while the localization of measurement is provided by the selection of $A$ in the range from 1 to 431,680 . Thus, in the text and figures below we omit the subscript $A$, but always indicate the size of $A$ in figure captions.

\subsection{GPU data processing}

GPU-based systems can significantly accelerate image processing. However, the specific implementation of computing schemes can considerably affect the final result. Namely, non-optimal handling of massive data can disavow the benefits of parallel computing. In the case we consider, the conventional organization of LSI data in the form of sequence of frames is very suitable for the real-time speckle contrast/flow calculations, but not optimal for the rhythm analysis since one needs to extract data point-by-point from all the frames. Another important issue arises from the need to exchange data between the computer RAM and graphics device memory.

To avoid the performance loss due to the above reasons, we put all the data to be processed into device memory (6 Gb for NVIDIA Tesla C2075) once for each program run (Fig. 1). Then, both spatial and temporal domains are selected for analysis interactively and only the resulted data are passed back to computer memory.

According to our tests performed on algorithms we use, GPU-based computations using such Nvidia devices as GeForce Titan, GeForce 650M, Tesla C2075 provide more then 200 times acceleration comparing with CPU (single core) program runs on 


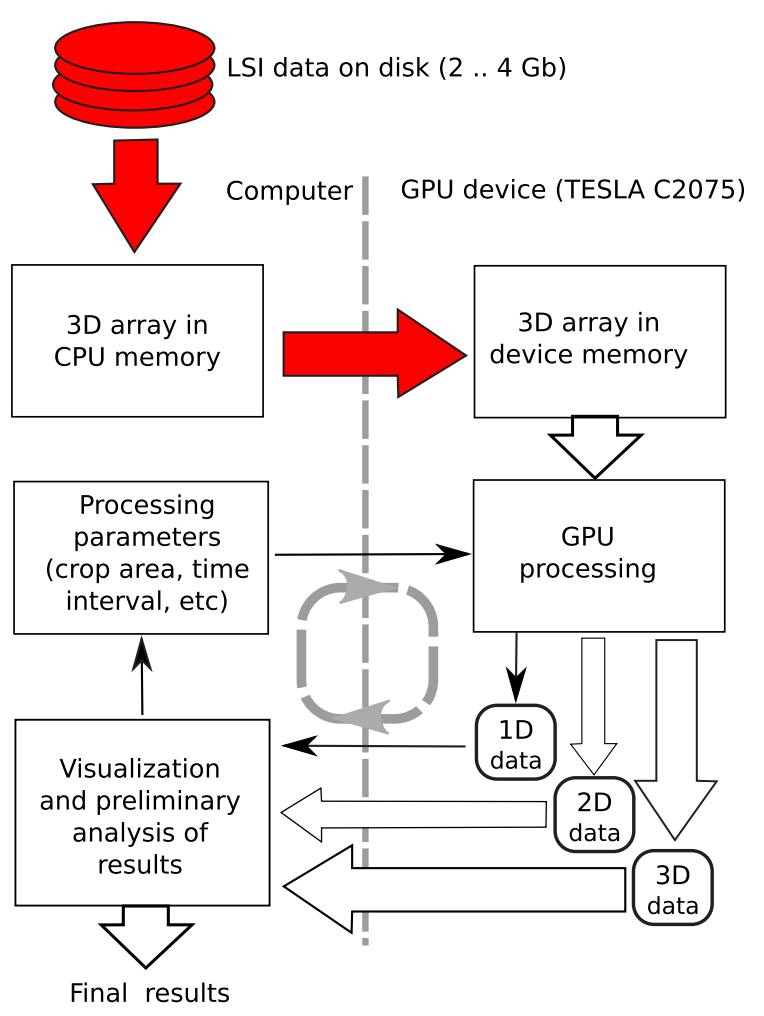

Fig. 1. A schematic illustration of GPU-based processing of large LSI data. The thicker arrows correspond to larger amount of data traffic between CPU memory on motherboard and memory on GPU unit. Red color denotes the data exchange that is done once for each program run.

Intel Core i7-3970X. For example, 1300 frames of $500 \times 400$ pixels each can be processed by simple (not fastest) wavelet transform algorithm in about $20 \mathrm{~s}$.

\section{Results}

While the application of windowed Fourier transform or wavelet transform to $1 \mathrm{D}$ data is straightforward, the complete transformation of 3D LSI data produces $4 \mathrm{D}$ array of results which is quite difficult to analyze. To keep the analysis reasonably simple, we use the following scheme:

- Calculate the spatially averaged power (Fourier or wavelet) spectrum over all data array. It delivers a simple $1 \mathrm{D}$ graph and allows one to estimate the most pronounced rhythms and to define the frequency range of interest, but information about spatial patterns is lost.

- Perform analysis for each selected frequency range (with averaging over frequencies within range) but keeping available spatial resolution. The result is $3 \mathrm{D}$ data being a sequence of frames showing the spatial structure and its evolution for the selected rhythm.

- Select small areas of interest and perform WFT or WT in wider frequency range with spatial averaging over selected areas. This gives $2 \mathrm{D}$ data (power vs time and frequency) that describe time evolution and possibly the frequency deviation at selected characteristic locations.

\subsection{Detection and localization of rhythmic activity}

In this section, we illustrate how the above described technique works when applied to the surrogate data. First, we test its ability to visualize a spatial pattern that is stable in time, but has slightly different frequency of one of the six rhythms as described in Sec. 2.1. Figure 2 shows representative snapshots.

The top and bottom rows are calculated at noise amplitudes of $50 \%$ and $200 \%$ of signal amplitude, respectively. Left panels (a) and (d) show the averaged flow data. No spatial structure is detectable in both cases. Central panels (b) and (e) display the spectral components of Fourier power spectrum at $f=0.30468$, while right panels (c) and (f) represent the power wavelet spectrum at $f=0.30$.

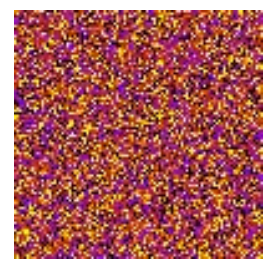

(a)

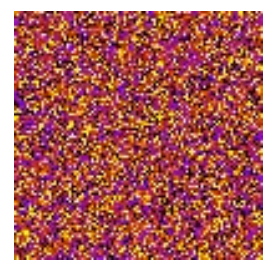

(d)

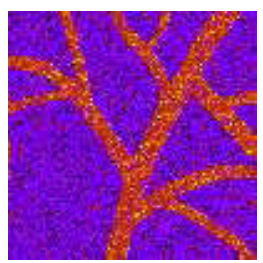

(b)

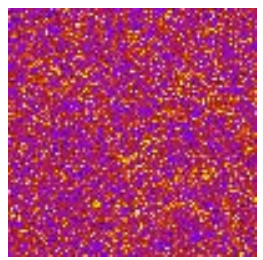

(e)

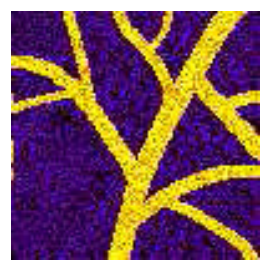

(c)

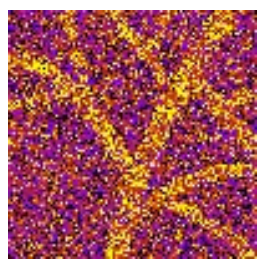

(f)
Fig. 2. Localization of rhythmic activity in a specific frequency domain. Surrogate data, $100 \times 100$ pixels. Oscillations with the period of $30 \mathrm{~s}$ are attributed to the tree-like object. Top and bottom rows are obtained at noise amplitude of $50 \%$ and $200 \%$ of signal amplitude, respectively. (a) and (d) shows time averaged LSI data; (b) and (e) Fourier power spectrum at frequency $f=0.30468$; (c) and (f) WT spectral power at frequency $f=0.30 . N_{A}=1$. 
(a)

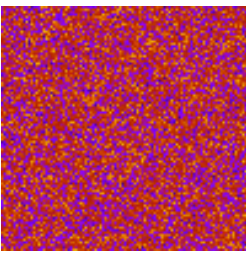

$\mathrm{t}=6$

(b)

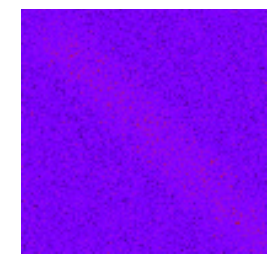

(c)

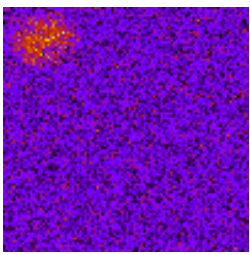

$0-256$

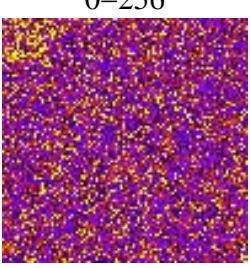

$\mathrm{t}=6$

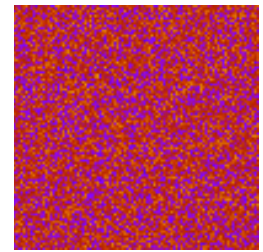

$\mathrm{t}=500$
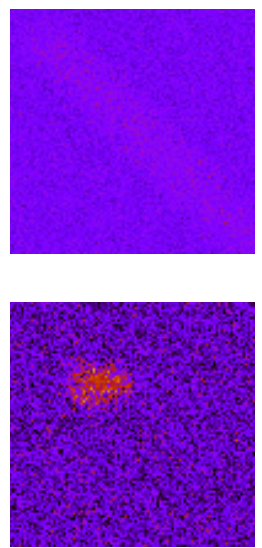

$512-756$

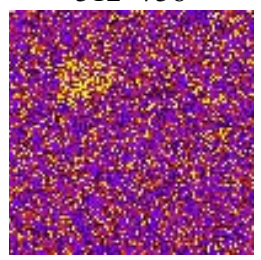

$\mathrm{t}=500$

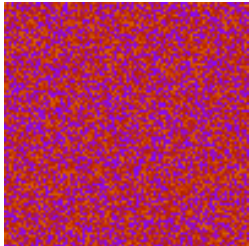

$t=1000$
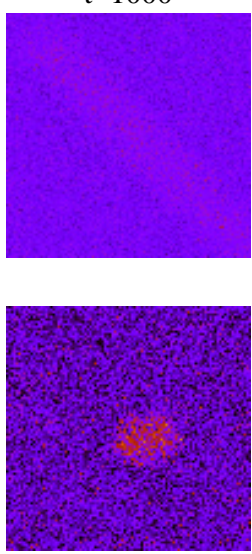

$1024-1280$

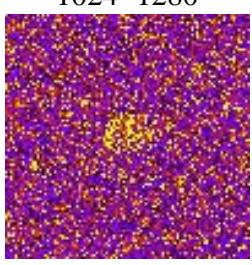

$t=1000$

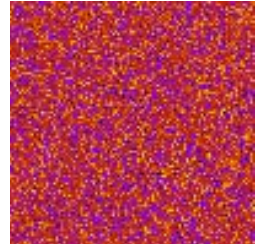

$\mathrm{t}=1500$
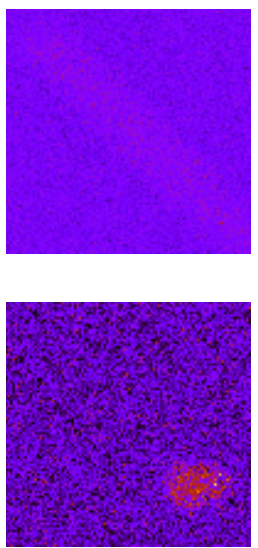

$1536-1792$

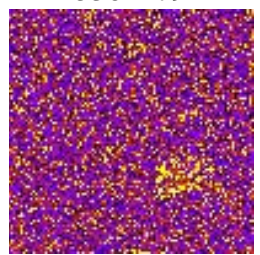

$\mathrm{t}=1500$

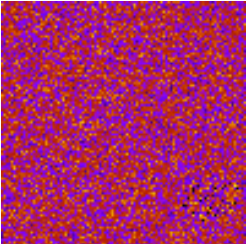

$\mathrm{t}=1840$
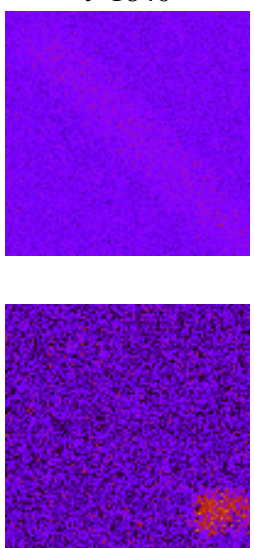

$1792-2048$

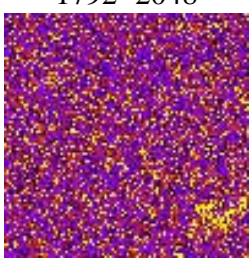

$\mathrm{t}=1840$

Fig. 3. On detection of a non-stationary (moving) rhythm source. Surrogate data. Row (a) — Surrogate LSI data; Row (b) — averaged Fourier power spectrum at $f=0.30468$ (the same for the all time moments); (c) and (d) show the selected frames of windowed Fourier power spectrum at the same frequency $f=0.30468$, and the wavelet power spectrum at $f=0.30$ for the indicated moments of time, respectively. $N_{A}=1$.

It is clear that at $50 \%$ noise level both methods successfully reveal the spatial structure hidden in a noisy and multimode signal. However, $200 \%$ noise level completely smears the structure (e) while the wavelet-based pattern is still visible (f).

In order to simulate a non-stationary rhythm source, we use the moving pattern in the form of a small spot that travels from top left corner of the field to the bottom right one (Fig. 3). With such choice, we also cover the case when rhythm source is activated or deactivated during the observation time. Four rows of the figure show the simulated flow image, spectral component $f=0.30468$ of time-averaged Fourier power spectrum, WFT at the same frequency, and the power wavelet spectrum at $f=0.30$ for indicated time intervals.

As in the previous example, the original data does not show any sign of the pattern. In the second row one can see the smeared stripe, both the third
(WFT-based analysis) and fourth (WT-based analysis) rows clearly show current location of the object.

In conclusion, the described technique successfully reveals both stationary and non-stationary spatial patterns formed by rhythmic modulation of LSI data.

\subsection{Spatio-temporal patterns in renal blood flow}

Figure 4(a) shows the optical image of a rat's kidney. The kidney is outlined by dashed curves. Numbers I-IV denote the regions of interest that we characterize in Fig. 7. The light spot in the centralleft part of the image consists of fat.

Panel (b) of the same figure shows the flow image averaged over recording time (44 min). The brightest parts (yellow) corresponds to relatively 


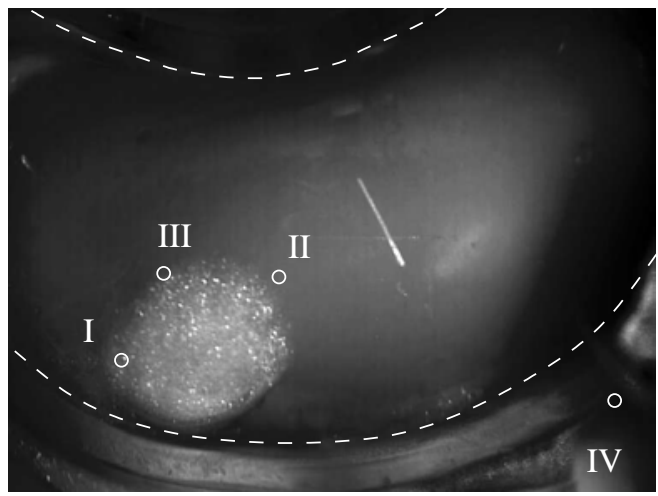

(a)

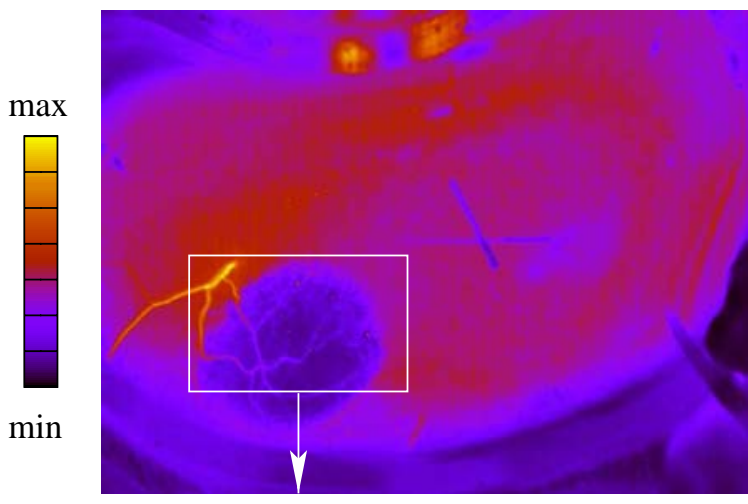

(b)

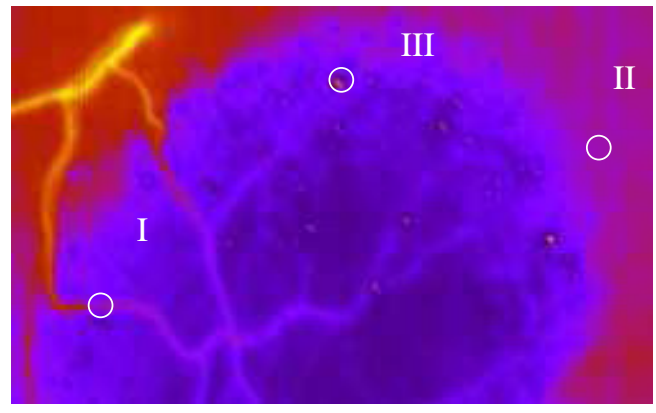

(c)

Fig. 4. Experimental data on kidney blood flow. (a) Optical image of rat's kidney (outlined by dashes) with marked points of interest. (b) Blood perfusion image, estimated from LSI and averaged over $44 \mathrm{~min}$. (c) Close up on the rectangle in (b).

large blood vessels, while the main part of the kidney surface is red. Low flow values (blue) correspond to the points outside the kidney. The precise location of points I, II, and III is shown in panel (c).

Figure 5 shows the averaged power spectra obtained according to (5) and averaged over time and space domains. There is a pronounced peak at $f=0.0185 \mathrm{~Hz}$ in both Fourier and wavelet spectra.

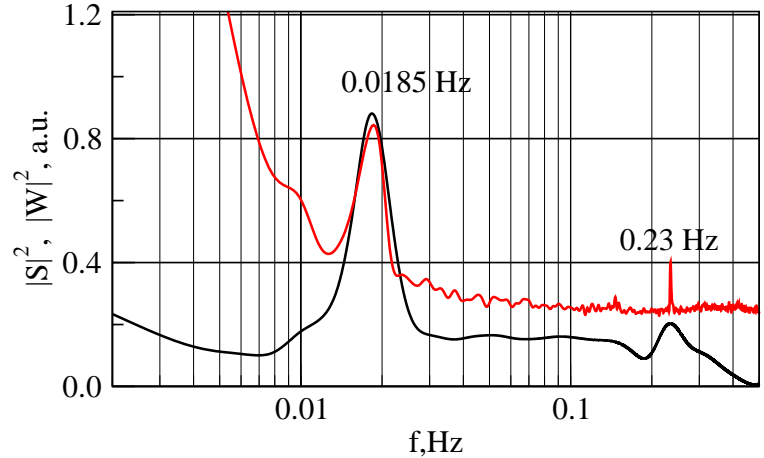

Fig. 5. Experimental data on kidney blood flow. Fourier (red) and wavelet (black) power spectra both obtained by averaging over space and time. Peaks at $f=0.0185 \mathrm{~Hz}$ and $f=0.23 \mathrm{~Hz}$ corresponds to the TGF and to the myogenic response. $N_{A}=431680$.

We associate this peak with TGF oscillations. This process modulates the arteriolar blood flow and thus can be detected by LSI. The smaller spectral peak at $f=0.23 \mathrm{~Hz}$ presumably associated with myogenic regulation in afferent arterioles of nephrons. This peak is considerably wider in wavelet spectrum. This can be explained by the used value of $k_{0}=1$ which is optimal for lower frequencies. Below, we focus exclusively on TGF rhythm and wavelet-based power spectra.

According to the above proposed scheme of rhythm analysis, next stage was focused on spatial patterns of the selected rhythmic activity. Figure 6

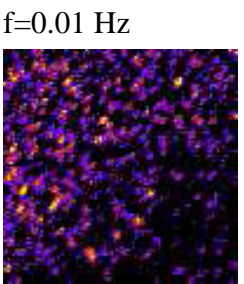

$\mathrm{f}=0.0184 \mathrm{~Hz}$

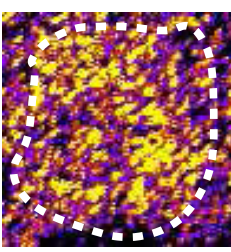

$\mathrm{t}=400 \mathrm{~s}$
0.2
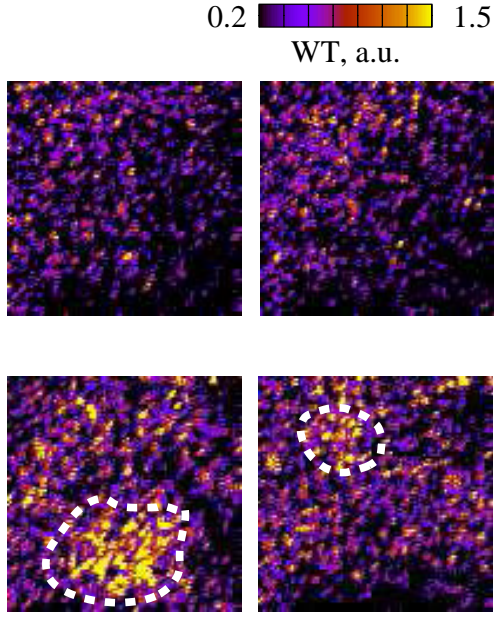

$\mathrm{t}=800 \mathrm{~s}$

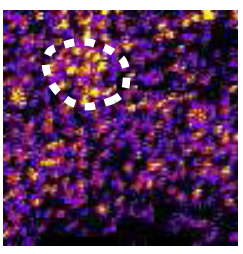

$\mathrm{t}=1200 \mathrm{~s}$
Fig. 6. The representative snapshots of WT power of the kidney segment recorded at $f=0.01 \mathrm{~Hz}$ (slow unspecified rhythm) and at $f=0.0185 \mathrm{~Hz}$ (TGF regulation). Dashed contour outlines the localized area of pronounced TGF rhythm. The time distance between frames corresponds to 8-9 TGF periods. $N_{A}=1$. 
shows the representative snapshots of wavelet based spectral power on $f=0.01 \mathrm{~Hz}$ (top row), and on TGF frequency $f=0.0185 \mathrm{~Hz}$ (bottom row). Low frequency activity at $\approx 0.01 \mathrm{~Hz}$ does not correspond to a spectral peak in Fig. 5 and is used as kind of reference pattern.

One can see that spatial distribution of this rhythm is remarkably stable. In contrast, three panels in bottom row demonstrate the pronounced temporal evolution: the spatial pattern of TGF oscillations changes with time. Considering the snapshots for $t=400,800$, and $1200 \mathrm{~s}$, the area of high activity (outlined by dashed curve) first shrinks to the spot, and then moves upward.
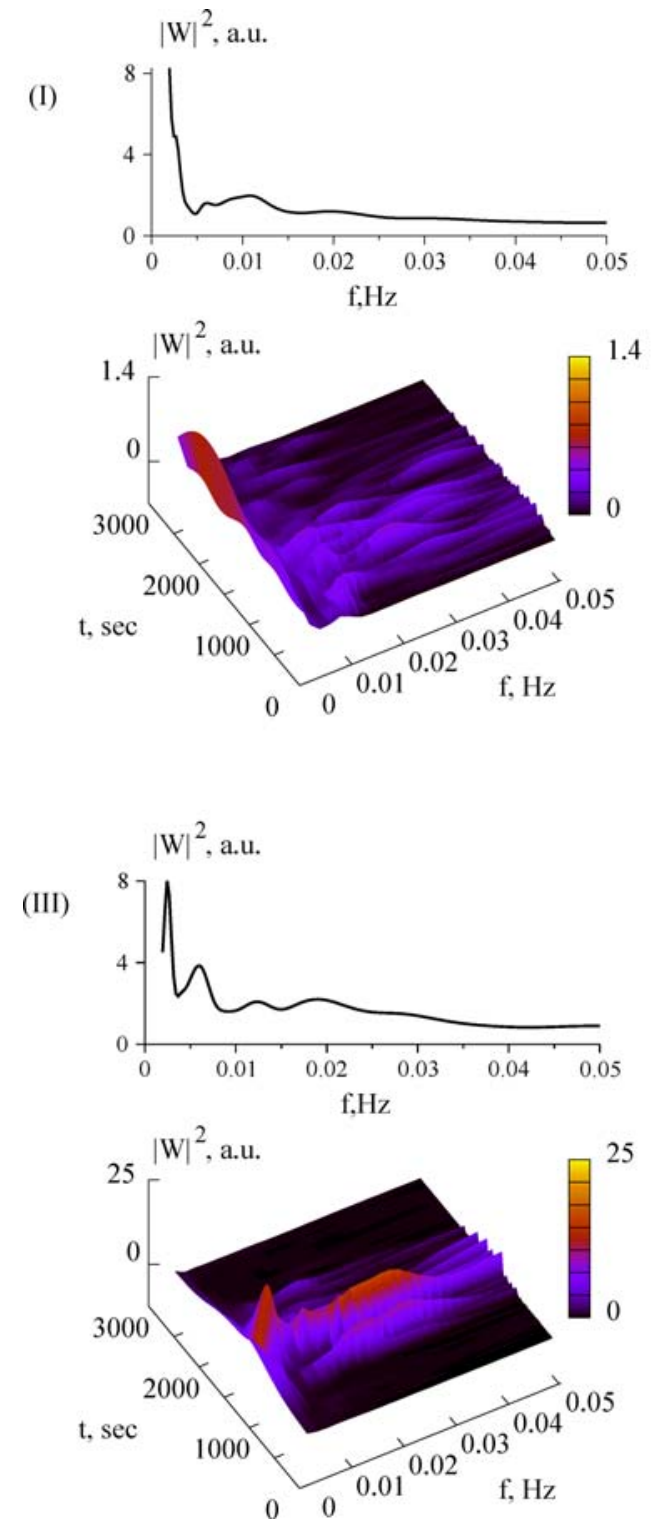

The time interval between snapshots corresponds to 7-8 periods of oscillations on TGF frequency. Thus, the observed behavior suggests that individual cortical nephrons oscillate not all the time, but rather show the modulated activity with a slow varying envelope.

The observation of averaged (Fig. 5) and evolving (Fig. 6) activity provides the basis to select the representative locations, that are numbered as I-IV in Fig. 4(a).

Namely, location I corresponds to a blood vessel segment, location II is in the region of high TGF activity, location III points to the small active zone that need to be identified, and location IV was

(II)
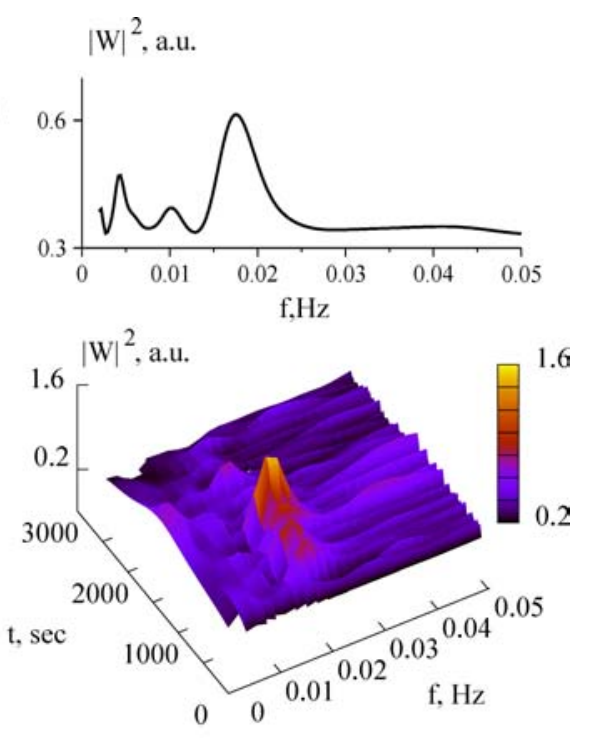

(IV)
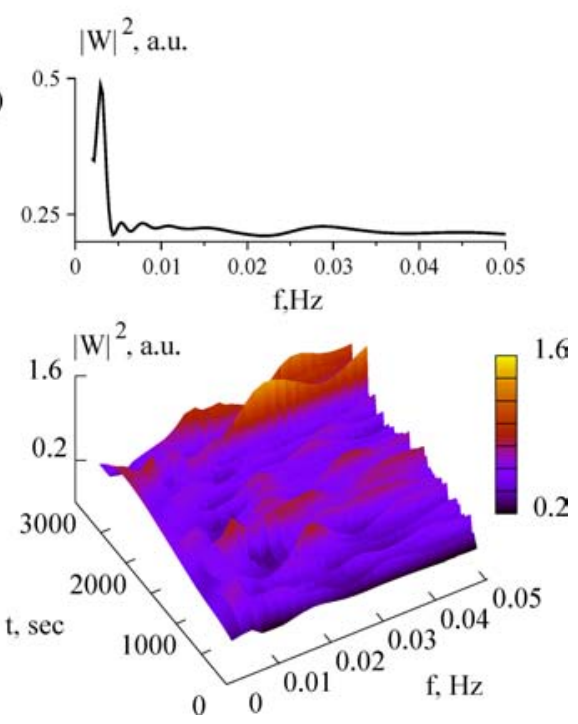

Fig. 7. Patterns of rhythmic activity in different regions of interest as indicated in Fig. 4 (a). $N_{A}=25$. 
selected out of kidney in order to provide a negative control, since no kidney-related activity is expected here. The detailed wavelet-based description of dynamics in regions of interest is given in Fig. 7. For each region of interest a small area of 25 pixels is selected to calculate both time-averaged and timedependent power spectra.

Region I, identified as a blood vessel, shows the maximal spectral power at very low frequencies. In the average spectrum, a smeared peak around $f=$ $0.01 \mathrm{~Hz}$ is seen, while almost all spectral power is concentrated near the origin, representing the closeto-consant flow component. In the time domain, this low frequency activity seems to be the most persistent in time, while the higher-frequency activity appears uncorrelated in time. The absence of TGF rhythm here is consistent with what is expected for a blood vessel. Note, due to low sampling rate $(1 \mathrm{~Hz})$ the data are free from fast changes of systemic arterial pressure, such as heart rate.

Region II corresponds to the pronounced rhythmic modulation of blood flow in nephrons. Peak at $f \approx 0.018 \mathrm{~Hz}$ is dominant but two more peaks at $f=0.01 \mathrm{~Hz}$ and $f=0.005 \mathrm{~Hz}$ are present, while the very low frequency components are absent. The inspection of temporal changes of activity shows, that the identified rhythms have a time-dependent amplitude but stable enough frequency.

Region III represents the small bright spot of $\approx 20$ pixels on the averaged flow map Fig. 4 (b). The averaged spectrum seems to be some mixture of one for the regions I and II, but the inspection of temporal dynamics reveals drastic differences. Namely, there is one but powerful burst of activity $\approx 800$ and $\approx 2000 \mathrm{~s}$. The shape of wavelet power spectrum at this time shows the presence of almost all frequencies in the analyzed range. Such all-frequency bursts in wavelet transform often correspond to the sharp rise or fall of the measured quantity or other signal discontinuity.

Lastly, region IV is placed outside of the kidney in order to verify the relevance of rhythmic components in locations I-II to the kidney autoregulation processes. First, note the relatively low spectral power recorded here. No activity is found in the TGF frequency range, while the single pronounced peak in the averaged spectrum is located at $\approx 0.003 \mathrm{~Hz}$. It corresponds to the period more than $5 \mathrm{~min}$ and can be hypothetically related to mechanical displacement of animal preparation. Thus, the spectral components at the same frequency in other regions of interest can also be regarded as artifacts.

\section{Conclusion}

LSI technique combined with spectral-temporal analysis of rhythmic activity becomes a powerful tool for monitoring of blood flow.

Technically, it can be done using existing methods such as windowed Fourier transform and continuous wavelet transform. The GPU-based parallel computing can provide a balanced solution for fast interactive processing of LSI data at reasonable costs.

Spectral-temporal analysis provides extensive output (for example, wavelet transform of $3 \mathrm{D}$ data results in $4 \mathrm{D}$ output). We propose the following approach to handle the data:

- To estimate the most pronounced rhythms and to define the frequency ranges of interest.

- To analyze each selected frequency range with available spatial resolution. The resulted 3D data show the spatial structures and their evolution for the selected rhythm.

- To select the specific regions of interest and to perform the spectral-temporal analysis with spatial averaging.

We have applied the proposed approach both to surrogate data and to experimentally recorded signal from the surface of rat's kidney.

Experiments with surrogate data demonstrated that the method can extract the information about oscillating patterns at high noise levels, when conventional (flow-based) analysis fails, and can detect a moving source of rhythm.

The analysis of kidney data allowed us to detect and to localize TGF dynamics arising from autoregulation processes in individual nephrons. Localized (averaged over small areas) analysis allows one to distinguish between TGF-active zones and TGFfree ones. This provides a basis for further development of automatized system for LSI-based kidney mapping.

Concerning computational aspects of our work, the usage of GPU devices such as Tesla C2075 or similar allows interactive search and analysis of rhythmic components. When computation time is of the same order with time needed by researcher to analyze the obtained result and plan the next step, one can say about "online post processing" of data, it is practically impossible with CPU-based 
computations which can take hours. Specifically, the calculation of wavelet transform as shown in Fig. 7, panels I-IV takes $4.5 \mathrm{~s}$ each. The one-frequency analysis of $100 \times 100$ pixels area takes $8.5 \mathrm{~s}$.

The evident next step would be the implementation of online rhythm analysis along with speckle contrast calculation. However, this task has its own specificity and is out of the present study.

Further development of this approach might be also focused on utilization of phase component of wavelet transform. Concerning the kidney data, our results show no pronounced phase coherent patterns of the TGF rhythm. This is consistent with recent theoretical predictions, ${ }^{17,18}$ but not suitable for testing of the data analysis method.

\section{Acknowledgments}

Authors would like to express his gratitude to N.-H. Holstein-Rathlou and O. Sosnovtseva (Univ. of Copenhagen, Denmark) for providing experimental data and useful discussions. D.E.P. acknowledges the support of Britton Chance Center for Biomedical Photonics, Wuhan National Laboratory for Optoelectronics, Huazhong University of Science and Technology, China.

\section{References}

1. M. Draijer, E. Hondebrink, T. van Leeuwen, W. Steenbergen, "Review of laser speckle contrast techniques for visualizing tissue perfusion", Lasers Med Sci. 24, 639 (2009).

2. J. D. Briers, "Laser speckle contrast imaging for measuring blood flow", Opt Appl. XXXVII(1-2), 139 (2007).

3. H. Cheng, Y. Yan, T. Q. Duong, "Laser speckle imaging of rat retinal blood flow with hybrid temporal and spatial analysis method", Proc. SPIE 7163, Ophthalmic Technologies XIX, 716304, doi: $10.1117 / 12.809906$.

4. M. A. Vilensky, O. V. Semyachkina-Glushkovskaya, P. A. Timoshina, J. V. Kuznetsova, I. A. Semyachkin-Glushkovskii, D. N. Agafonov, V. V. Tuchin, "Laser speckle-imaging of blood microcirculation in the brain cortex of laboratory rats in stress", Quantum Electron. 42, 489 (2012).

5. J. K. Meisner, S. Sumer, K. P. Murrell, T. J. Higgins, R. J. Price, "Laser speckle flowmetry method for measuring spatial and temporal hemodynamic alterations throughout large microvascular networks", Microcirculation. 19, 619 (2012).
6. N. H. Holstein-Rathlou, O. V. Sosnovtseva, A. N. Pavlov, W. A. Cupples, C. M. Sorensen, D. J. Marsh, "Nephron blood flow dynamics measured by laser speckle contrast imaging", Am. J. Physiol. Renal. Physiol. 300, F319 (2011).

7. W. Luo, P. Li, Z. Wang, S. Zeng, Q. Luo, "Tracing collateral circulation after ischemia in rat cortex by laser speckle imaging", J. Innov. Opt. Health Sci. 1 (2), 217 (2008).

8. P. Zakharov, A. C. Vlker, M. T. Wyss, F. Haiss, N. Calcinaghi, C. Zunzunegui, A. Buck, F. Scheffold, B. Weber, "Dynamic laser speckle imaging of cerebral blood flow", Opt. Express. 17(16), 13904 (2009).

9. Q. Luo, C. Jiang, P. Li, H. Cheng, Z. Wang, Z. Wang, V. V. Tuchin, "Laser speckle imaging of cerebral blood flow ", Handbook of CoherentDomain Optical Methods: Biomedical Diagnostics, Environmental Monitoring, and Materials Science, Chap. 5, V. V. Tuchin, Ed., pp. 167-211, Springer Science + Business Media, New York (2013).

10. A. Colantuoni, S. Bertuglia, M. Intaglietta, "Quantitation of rhythmic diameter changes in arterial microcirculation", Am. J. Physiol. 246, H508-H517 (1984).

11. I. S. Bartlett, G. J. Crane, T. O. Neild, S. S. Segal, "Electrophysiological basis of arteriolar vasomotion in vivo", J. Vasc. Res. 37, 568 (2000).

12. H. Nilsson, C. Aalkjaer, "Vasomotion: Mechanisms and physiological importance", Mol. Interv. 3, 79 (2003).

13. C. Aalkjaer, H. Nilsson, "Vasomotion: cellular background for the oscillator and for the synchronization of smooth muscle cells". Br. J. Pharmacol. 144, 605 (2005).

14. N.-H. Holstein-Rathlou, P. P. Leyssac, "TGFmediated oscillations in the proximal intratubulular pressure: Differences between spontaneously hypertensive rats and Wistar-Kyoto rats", Acta Physiol. Scand. 126, 333 (1986).

15. N.-H. Holstein-Rathlou, D. J. Marsh, "Renal blood flow regulation and arterial pressure fluctuations: a case study in nonlinear dynamics", Physiol. Rev. $\mathbf{7 4}$ 637 (1994).

16. A. H. Oien, K. Aukland, "A multinephron model of renal blood flow autoregulation by tubuloglomerular feedback and myogenic response", Acta Physiol. Scand. 143, 71 (1991).

17. D. D. Postnov, D. E. Postnov, D. J. Marsh, N.-H. Holstein-Rathlou, O. V. Sosnovtseva, "Dynamics of nephron-vascular network", Bull. Math. Biol. 74 (12), 2820 (2012).

18. D. J. Marsh, A. S. Wexler, A. Brazhe, D. E. Postnov, O. V. Sosnovtseva and N. H. Holstein-Rathlou, "Multinephron dynamics on the renal vascular 
network", Amer. J. Physiol. — Renal Physiol. 304(1) F88-F102 (2013).

19. S. Mallat, A Wavelet Tour of Signal Processing, Third Edition: The Sparse Way, Academic Press, Burlington, MA (2009).

20. R. Bracewell, The Fourier Transform and Its Applications, McGraw-Hill Science/Engineering/Math, New York (1999).

21. P. S. Addison, The Illustrated Wavelet Transform Handbook: Introductory Theory and Applications in Science, Engineering, Medicine and Finance, Institute of Physics Publishing, London, UK (2002).

22. J. Sanders, E. Kandrot, CUDA by Example: An Introduction to General-Purpose GPU Programming, Addison-Wesley Professional, Cambridge (2010).

23. W.-M W. Hwu, GPU Computing Gems Emerald Edition (Applications of GPU Computing Series),
Morgan Kaufmann Publishers Inc., San Francisco, CA, USA (2011).

24. K. K. C. Lee, A. Mariampillai, J. X. Z. Yu, D. W. Cadotte, B. C. Wilson, B. A. Standish, V. X. D. Yang, "Real-time speckle variance swept-source optical coherence tomography using a graphics processing unit", Biomed. Opt. Express. 3(7), 1557 (2012).

25. S. Liu, P. Li, Q. Luo, "Fast blood flow visualization of high-resolution laser speckle imaging data using graphics processing unit", Opt. Express. 16(19), 14321 (2008).

26. O. Yang, D. Cuccia, B. Choi, "Real-time blood flow visualization using the graphics processing unit", J. Biomed. Opt. 16(1), 016009 (2011). 